\title{
Clinical and biological features of mantle cell lymphoma patients with co-expression of CD10 and BCL-6: a retrospective study
}

\author{
Wanzi Chen ${ }^{1}$, Hangmin Liu ${ }^{2}$, Pengcheng Wang ${ }^{3}$, Guoping $\mathrm{Li}^{3}$ \\ ${ }^{1}$ Fujian Institute of Hematology, Fujian Provincial Key Laboratory on Hematology, Fujian Medical University Union Hospital, Fuzhou, China; \\ ${ }^{2}$ Clinical Laboratory, Fujian Medical University Union Hospital, Fuzhou, China; ${ }^{3}$ Department of pathology, First Affiliated Hospital of Fujian \\ Medical University, Fuzhou, China \\ Contributions: (I) Conception and design: W Chen; (II) Administrative support: W Chen; (III) Provision of study materials or patients: W Chen, H \\ Liu; (IV) Collection and assembly of data: W Chen, H Liu, P Wang; (V) Data analysis and interpretation: W Chen, G Li; (VI) Manuscript writing: \\ All authors; (VII) Final approval of manuscript: All authors. \\ Correspondence to: Guoping Li. Department of pathology, First Affiliated Hospital of Fujian Medical University, 20 Chazhong Road, Fuzhou 350004, \\ China. Email: liguoping_827@126.com.
}

Background: Mantle cell lymphoma (MCL) with co-expression of CD10 and BCL-6 was scarcely reported, and its biological features were largely remained unknown. Thus, this study aimed to describe the clinical and biological features, as well as outcome of MCL patients with co-expression of CD10 and BCL-6. Methods: A total of 104 cases of MCL who were admitted to our hospital between January 2011 and October 2018 were recruited. Those patients were diagnosed according to the 2016 World Health Organization (WHO) classification system for tumors of the hematopoietic and lymphoid tissues, in compliance with the results of cytomorphology and immunohistochemical analysis. Patients were followed up through telephone interviews, medical records. Differences in age, gender, leukocyte count, lactate dehydrogenase (LDH) level, beta-2 microglobulin ( $\beta 2-\mathrm{MG}$ ) level and results of immunohistochemistry were analyzed. Then the event-free survival (EFS) rate and overall survival (OS) rate were performed by the Kaplan-Meier method and log-rank test.

Results: The results showed that, in total, 5 patients had superficial lymph node enlargement, 2 patients suffered from abdominal discomfort, and 1 patient's red blood cell (RBC) count was abnormal at the time of diagnosis. All patients were in stage IV, $75 \%$ had bone marrow involvement, and $75 \%$ and $100 \%$ of patients had elevated levels of $\mathrm{LDH}$ and $\beta 2-\mathrm{MG}$, respectively. Three classic genes and five variants were involved in the 8 patients. MCL patients with the co-expression of CD10 and BCL-6 had higher Ki-67 index, White blood cell (WBC) count, LDH level, and $\beta 2-M G$ level than those of without co-expression of CD10 and BCL-6 ( $\mathrm{P}=0.025,0.038,0.015$, and 0.021, respectively). Besides, MCL patients with CD10 and BCL-6 co-expression had shorter OS and EFS $\left(\chi^{2}=6.401\right.$ and $5.975 ; \mathrm{P}=0.011$ and 0.015 , respectively), indicating patients' susceptibility to get complex karyotype and TP53 abnormality.

Conclusions: MCL patients with co-expression of CD10 and BCL-6 were more likely to have bone marrow involvement, higher Ki-67 index, increased WBC count, and elevated levels of LDH and $\beta 2-\mathrm{MG}$ at the time of diagnosis, then might has complex cytogenetic and poor prognosis.

Keywords: Mantle cell lymphoma (MCL); CD10; BCL-6; immunohistochemistry; cytogenetic factors

Submitted May 22, 2021. Accepted for publication Sep 03, 2021.

doi: $10.21037 /$ tcr-21-882

View this article at: https://dx.doi.org/10.21037/tcr-21-882 


\section{Introduction}

Mantle cell lymphoma (MCL) is an aggressive mature B-cell lymphoma that is caused by juxtaposition of CCND1 located at chromosome $11 \mathrm{q} 13$ with the promoter region of immunoglobulin heavy-chain (IGH) at chromosome $14 \mathrm{q} 32$, resulting in overexpression of cyclinD1. The CCND1/IGH translocation is a genetic abnormality, which may occur in $>90 \%$ of MCL cases, and it is mainly detected by conventional karyotyping or fluorescence in situ hybridization (FISH) $(1,2)$. This translocation was believed to be a primary oncogenic event in MCL $(3,4)$. Patients with MCL have been often treated with intensive chemoimmunotherapy at the time of diagnosis (5). However, it was mainly incurable in the majority of patients, which could ultimately result in relapse, and disease progression (DP) (6,7). Therapies for MCL have classically been envisioned around the target of obtaining longer remission by deeper initial responses using intensive induction regimens. Despite the uniform morphology and immunophenotype of MCL, clinical and biological heterogeneity may be observed in MCL cases. The socalled aberrant phenotypes have been described, CD5 negative-MCL, CCND1 negative-MCL etc. However, further researches are required to explore the biological mechanism of MCL more effectively, and to establish a more reliable diagnostic system for MCL.

The majority of cases with MCL were found to arise from a naïve pre-germinal center (GC) B cell, and the majority of MCL cases had a characteristic immunophenotype, which was positive for B cell antigens (CD5, BCL-2, and cyclinD1), and negative for CD23 and follicular center cellassociated antigens, such as CD10 and BCL-6. A number of cases with aberrant immunophenotypes have been reported in recent years. CD10 is expressed on hematogones (normal precursor B cells in bone marrow), normal GC B cells, and neoplastic conditions $(8,9)$, and CD10 gene expression plays a role in the pathogenesis of diffuse large B-cell lymphoma. Therefore, CD10 could contribute to distinguish MCL from other CD10-positive B cell lymphomas, including follicular lymphoma. During the humoral response, BCL6 functions as a master regulator of the GC B cell phenotype. GCs emerge in the secondary lymphoid organs upon B cell activation and provide the setting for massive clonal expansion, immunoglobulin somatic hypermutation, and class switch recombination, leading to antibody affinity maturation $(10,11)$. Among B-cell neoplasms, BCL-6 was found to be expressed by in follicular B-cell lymphomas, diffuse large B-cell lymphomas, and Burkitt lymphomas. To date, few cases of MCL with co-expression of CD10 and BCL-6 have been reported. The co-expression of CD10 and BCL-6 in MCL patients poses intriguing questions on its biological significance, while the majority of previously published studies have concentrated on single case reports (12-14). The biological features of MCL patients with coexpression of CD10 and BCL-6 were largely remained unknown. Hence, the present study aimed to investigate clinicopathological and cytogenetic features of MCL cases concerning the co-expression of CD10 and BCL-6.

We present the following article in accordance with the REMARK reporting checklist (available at https://dx.doi. org/10.21037/tcr-21-882).

\section{Methods}

\section{Patients}

A total of 104 cases with MCL who were admitted to the Fujian Medical University Union Hospital (Fuzhou, China) between January 2011 and October 2018 were recruited. There were 8 MCL patients with co-expression of CD10 and BCL-6 were identified Those patients were diagnosed according to the 2016 World Health Organization (WHO) classification system for tumors of the hematopoietic and lymphoid tissues, as well as being in compliance with the results of cytomorphology and immunohistochemical analysis (3). The study was conducted in accordance with the Declaration of Helsinki (as revised in 2013) and approved by the Ethics Committee of the Fujian Medical University Union Hospital (approval No. 2019KY123) and individual consent for this retrospective analysis was waived.

\section{Clinical data}

The following clinical data were collected: age, gender, disease stage, laboratory parameters [white blood cell (WBC) count, hemoglobin (Hb) level, platelet (PLT) count, lactate dehydrogenase $(\mathrm{LDH})$, and beta-2 microglobulin ( $\beta 2-\mathrm{MG})]$, immunohistochemical tumor markers (Ki-67, CD20, CD5, cyclinD1, BCL-2, BCL-6, and CD10), results of cytogenetic tests (karyotyping and FISH), and treatmentassociated factors. Imaging methods [B-mode ultrasound, computed tomography (CT), magnetic resonance imaging (MRI), and positron emission tomography-CT (PETCT)] were conducted. Routine karyotyping was performed with R-banding technique, and two FISH probes, IgH/ 
CCND1 and $p 53$ gene (Beijing Jin Bu Jia Biotechnology Co., Ltd., Beijing, China), were used to investigate the cytogenetic features of the samples. When IHH/CCND1 fusion occurred, two yellow fusion signals produced by the proximity or superposition of green and red nuclei, one red and one green (2Y1R1G) signals, were presented. Otherwise, two red and two green signals appeared (2R2G).

\section{Outcomes}

Chemotherapy is the preferred treatment for MCL patients. Therapeutic schemes mainly include rituximab plus hyperfractionated cyclophosphamide, vincristine, doxorubicin, and dexamethasone (R-Hyper-CVAD), rituximab with cyclophosphamide, doxorubicin, vincristine, and prednisone (R-CHOP), and other methods [CHOP, fludarabine plus cyclophosphamide (FC), cyclophosphamide, vincristine, and prednisone (COP), gemcitabine-oxaliplatin (GEMOX), etoposide, prednisone, vincristine, cyclophosphamide, and doxorubicin (EPOCH), etc.]. Tumor responses [complete response (CR), partial response (PR), and $\mathrm{DP}$ ] were evaluated according to the Response Evaluation Criteria in Solid Tumors (RECIST). Overall survival (OS) was defined as the time from the date of diagnosis to the date of death or the last follow-up, and event-free survival (EFS) was defined as the time from the diagnosis to disease progression or recurrence or death or the last follow-up.

\section{Follow-up}

To identify these outcomes, participants were followed over time using a variety of methods, including telephone interviews, medical examiner reports, and computer-based patient record until October 31, 2019.

\section{Statistical analysis}

Data were statistically processed using SPSS 18.0 software (IBM Corp., Armonk, NY, USA). The categorical data were expressed as percentage, and measured data were presented as mean \pm standard deviation. The Chi-square test was used for comparing differences between MCL patients with co-expression of CD10 and BCL-6 and MCL patients without co-expression of CD10 and BCL-6. Univariate and multivariate COX regression analysis was performed. Survival analysis was performed by the Kaplan-Meier method and log-rank test. We attempted to set the level of significance at $\mathrm{P}<0.05$ which is two-sided.

\section{Results}

\section{Clinical data}

Herein, 8 MCL patients with co-expression of CD10 and BCL-6 were identified from 104 MCL patients [male $(n=7)$ versus female $(n=1)$ ], with a mean follow-up of 12 months (range, 2-77 months). The patients' median age was 63 years old (range, 52-72 years old). In total, 5 patients (44\%) had superficial lymph node enlargement, 2 patients suffered from abdominal discomfort, and 1 patient's red blood cell (RBC) count was abnormal at the time of diagnosis. All patients were in stage IV, 75.0\% (6/8) had bone marrow involvement, and $75.00 \%(6 / 8)$ and $100 \%(8 / 8)$ of patients had elevated levels of $\mathrm{LDH}$ and $\beta 2-M G$, respectively. Three classic genes and five variants were involved in the 8 patients. The heteromorphism was dominated by nodular or diffuse growth, the nucleus was slightly larger than the classical one, and the nuclear chromatin was meticulous (Figure 1A). Ki-67-positive rate of tumor cells was in the range of $40-90 \%$ in all cases. Immunohistochemical assay showed CD20+ (8/8), CD5+ (7/8), cyclinD1+ (8/8), and BCL-2+ (6/7). The results of FISH displayed yellow IGH/CCND1 fusion signal (Y) (Figure 1B-1I). MCL patients with the co-expression of CD10 and BCL-6 had higher Ki-67 index, WBC count, $\mathrm{LDH}$ level, and $32-\mathrm{MG}$ level than those of without coexpression of CD10 and BCL-6 (P=0.025, 0.038, 0.015, and 0.021 , respectively). There were no significant differences among the remaining items $(\mathrm{P}>0.05)$ (Table 1).

\section{Cytogenetic features}

The results of routine karyotyping of 8 MCL cases with co-expression of CD10 and BCL-6 showed no split phase $(1 / 8)$, normal karyotype $(1 / 8)$, and $\mathrm{t}(11 ; 14)(\mathrm{q} 13 ; \mathrm{q} 32)(6 / 8)$, respectively. There were two complex karyotypes (CK) accompanied with other chromosomal abnormalities, including chromosomes 1, 5, 7, 10, 17, and 19. However, the result of FISH was positive in all the patients, and the result of TP53 genetic test was positive in $50 \%$ of the patients (3/6) (Table 2).

\section{Survival analysis}

Three patients withdrew treatment after diagnosis for the 

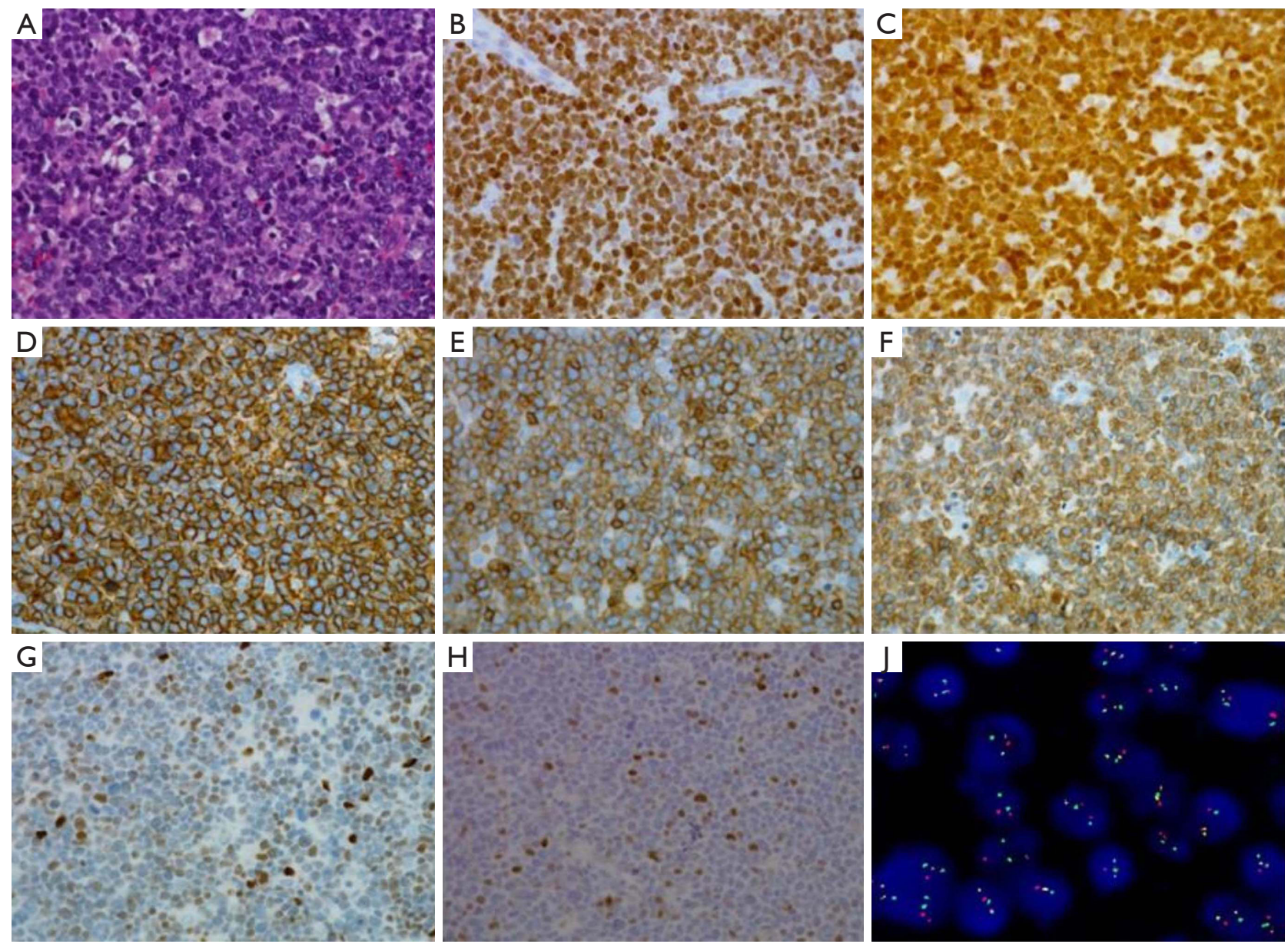

Figure 1 Immunohistochemical staining and FISH analyses of MCL. (A) HE staining, $\times 400$; (B-H) immunohistochemical staining of Ki67, CyclinD1, CD20, CD5, BCL-2, BCL-6, CD10, ×400; (I) FISH IgH/CCND1 fusion, 2Y1R1G, ×1,000. FISH, fluorescence in situ hybridization; MCL, mantle cell lymphoma.

Table 1 Clinical features of MCL with CD10and BCL-6 coexpression

\begin{tabular}{|c|c|c|c|}
\hline \multirow{2}{*}{ Characteristics } & \multicolumn{2}{|c|}{ CD10 and BCL-6 co-expression } & \multirow{2}{*}{$P$ value } \\
\hline & Yes $(n=8)$ & No $(n=87)$ & \\
\hline Age (year) & $61.75 \pm 7.13$ & $58.41 \pm 10.85$ & 0.397 \\
\hline Sex (male/female) & $7 / 1$ & $71 / 15$ & 0.684 \\
\hline Ki-67 (\%) & $63.13 \pm 19.45$ & $45.00 \pm 21.69$ & 0.025 \\
\hline WBC $\left(\times 10^{9} / \mathrm{L}\right)$ & $29.63 \pm 35.08$ & $13.58 \pm 19.05$ & 0.038 \\
\hline $\mathrm{Hb}(\mathrm{g} / \mathrm{L})$ & $106.63 \pm 27.25$ & $113.23 \pm 28.25$ & 0.527 \\
\hline $\operatorname{PLT}\left(\times 10^{9} / \mathrm{L}\right)$ & $133.38 \pm 73.77$ & $184.40 \pm 116.71$ & 0.239 \\
\hline LDH (IU/L) & $462.00 \pm 208.37$ & $299.99 \pm 174.96$ & 0.015 \\
\hline$\beta 2-M G(m g / L)$ & $6.34 \pm 2.51$ & $3.98 \pm 2.79$ & 0.021 \\
\hline Classic/variants & $3 / 5$ & $78 / 9$ & 0.001 \\
\hline
\end{tabular}

$\mathrm{P}<0.05$ was for having statistically significant difference. WBC, white blood cell; $\mathrm{Hb}$, hemoglobin; PLT, platelet; $\mathrm{LDH}$, lactate dehydrogenase; $\beta 2-\mathrm{MG}$, beta-2 microglobulin. economic reasons, and the others were mainly treated with chemotherapy-based regimens, including R-CHOP $(n=44)$, R-hyper-CVAD ( $\mathrm{n}=40)$, and other regimens $(\mathrm{n}=17)$. MCL patients with co-expression of CD10 and BCL-6 underwent R-CHOP ( $n=4)$, R-hyper-CVAD ( $n=2)$, COP $(n=1)$, and FC $(n=1)$. There were only 2 cases of CR, 1 case of $P R$, and 5 cases of DP who died after treatment. The median survival time (12 months) was significantly shorter in MCL patients with co-expression of CD10 and BCL- 6 than that in those without co-expression of CD10 and BCL-6 (54 months), and there were statistically significant differences in 1 -year OS rate $(16.7 \%$ vs. $88.9 \%)$ and 1 -year EFS rate $(16.7 \%$ vs. $66.9 \%)$ between the two groups $(\mathrm{P}=0.011$ and 0.015 , respectively) (Figure 2). Further, we evaluated the impact on survival and prognostic factors on gender, WBC counts, Ki-67 index, SOX11 $1^{+}$, co-expression 
Table 2 Cytogenetic results of MCL with CD10 and BCL-6 co-expression

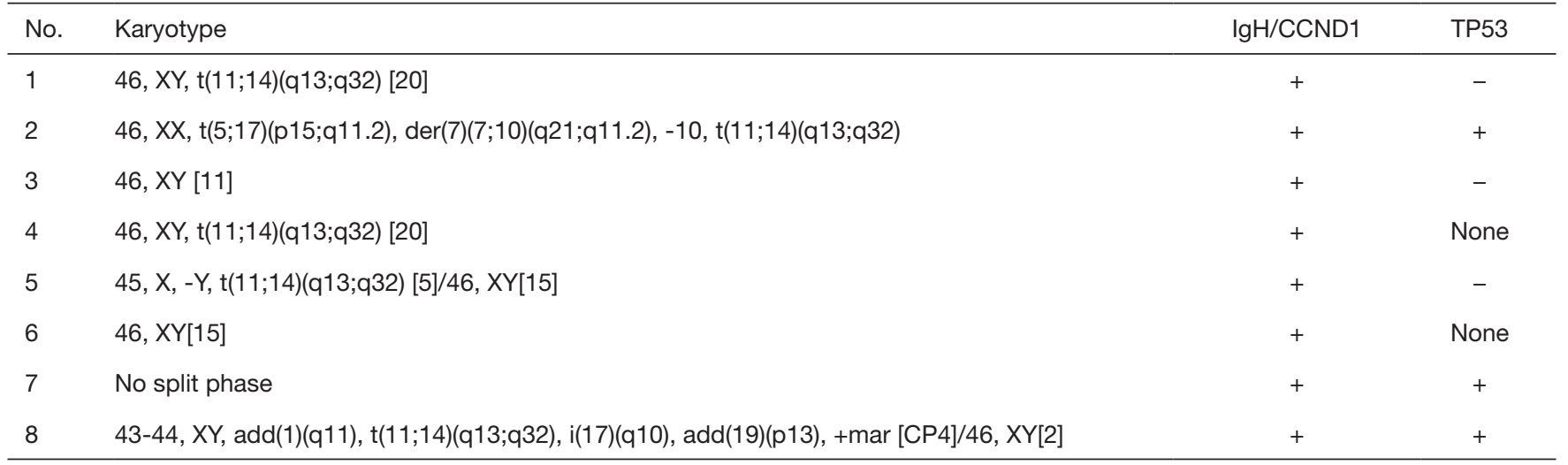

+, gene positive; -, gene negative. MCL, Mantle cell lymphoma.
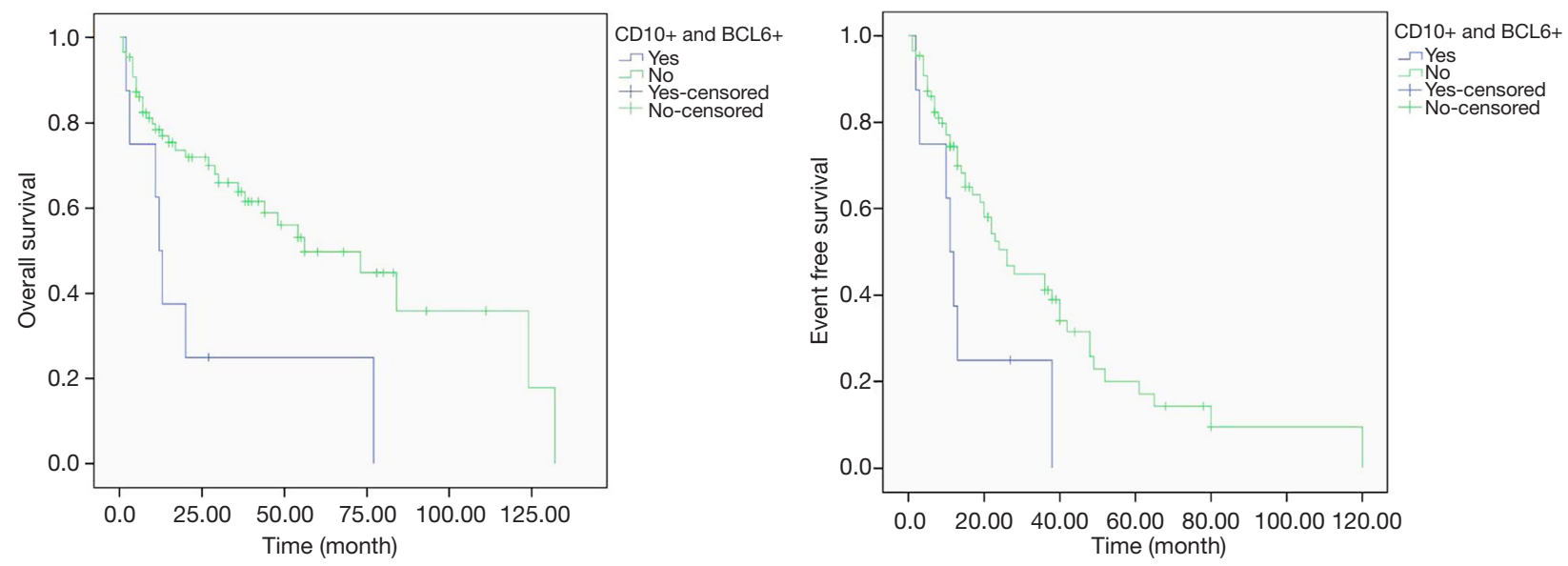

Figure 2 Survival analyses by Kaplan-Meier and log-rank.

of CD10 and BCL-6, LDH, $32-\mathrm{MG}$, CR for the four courses, bone-marrow involvement, and bone-marrow transplant by Cox regression analysis. The multivariate COX regression analysis results showed that WBC counts, SOX11 $1^{+}$, CR for the four courses had correlation with survival time $(\mathrm{P}=0.015,0.021$, and 0.08 respectively; $\mathrm{HR}=4.86,0.13,278.44$ respectively). Patients with coexpression of CD10 and BCL-6 had no correlation with survival time $(\mathrm{P}=0.173)$ (Table 3).

\section{Discussion}

MCL is an aggressive mature B cell lymphoma characterized by the $\mathrm{t}(11 ; 14)$ IGH-CCND1 translocation, leading to the high expression of cyclinD1 (15). MCL patients are typically treated with intensive chemoimmunotherapy regimens at the time of diagnosis with an inferior OS and a high risk of relapse in the majority of cases (7). Immunohistochemical staining showed that MCL cells were positive for CD20, CD5, cyclinD1, and BCL-2, while those cells were negative for CD10 and BCL-6 (16). Recent studies showed that MCL is not only associated with diverse morphological features, but also with aberrant expressions of immunophenotypic markers, such as GC markers (CD10 and BCL-6) $(17,18)$. A few scholars studied Immunophenotypic profile and clinical characteristics of patients with advanced stage MCL. To date, few cases of MCL with CD10 and BCL-6 co-expression were reported, and its biological mechanism remains elusive.

In the current study, $8 \mathrm{MCL}$ patients with CD10 and BCL-6 co-expression were mainly elderly men, accounting for $87.5 \%$. Patients were in stage IV, and their median 
Table 3 Multivariate Cox regression analyses for overall survival

\begin{tabular}{|c|c|c|}
\hline Characteristics & $\mathrm{HR}(95.0 \% \mathrm{Cl})$ & $P$ value \\
\hline WBC $\left(\times 10^{9} / \mathrm{L}\right)$ & & 0.015 \\
\hline$<15$ & 1.0 & \\
\hline$\geq 15$ & $4.864(1.353-17.489)$ & \\
\hline Ki-67 index (\%) & & 0.286 \\
\hline$<50$ & 1.0 & \\
\hline$\geq 50$ & $0.504(0.143-1.776)$ & \\
\hline Sox11 & & 0.021 \\
\hline Negative & 1.0 & \\
\hline Positive & $0.130(0.023-0.731)$ & \\
\hline $\mathrm{CD}_{10}{ }^{+}$and $\mathrm{BCL}-6^{+}$ & & 0.173 \\
\hline Yes & 1.0 & \\
\hline Not & $3.4221(0.583-20.067)$ & \\
\hline LDH & & 0.995 \\
\hline Normal & 1.0 & \\
\hline High & $1.005(.190-5.314)$ & \\
\hline$\beta 2-M G$ & & 0.523 \\
\hline Normal & 1.0 & \\
\hline High & $1.701(0.333-8.685)$ & \\
\hline CR in four courses & & 0.008 \\
\hline CR & 1.0 & \\
\hline Not CR & $278.436(4.233-18314.526)$ & \\
\hline Bone-marrow involvement & & 0.642 \\
\hline Yes & 1.0 & \\
\hline Not & $0.709(0.166-3.032)$ & \\
\hline
\end{tabular}

$\mathrm{P}<0.05$ was for having statistically significant difference. WBC, white blood cell; $\mathrm{Hb}$, hemoglobin; PLT, platelet; $\mathrm{LDH}$, lactate dehydrogenase; $\beta 2-\mathrm{MG}$, beta-2 microglobulin; $\mathrm{CR}$, complete response.

age was 63 years old (range, 52-72 years old) at the time of diagnosis, which was similar to the rate reported previously (19). These patients had a higher Ki-67 index $(63.13 \% \pm 19.45 \%)$ and were more likely to present with variants $(\mathrm{P}=0.001)$. Ki-67 index is used as a marker for determining the growth fraction of a given population of cells, and it has been widely used for assessing proliferation of malignant lymphomas (20). A study showed that (21), there was a significant correlation between $\mathrm{Ki}-67$ index and MCL subtype. Gao et al. (13) demonstrated that, in 4 of 50 (8\%) MCL cases, CD10 was expressed, and there was no significant difference in $\mathrm{Ki}-67$ rate between MCL with a variant immunophenotype and MCL with a typical immunophenotype. However, MCL carries a high mutational load that may originate from cells strongly influenced by the GC microenvironment. The co-expression of CD10 and BCL- 6 was scarcely reported in MCL patients. However, MCL variants were found more common to have aberrant expressions of CD10 and BCL-6 (22). Zanetto et al. (23) pointed out that aberrant expression of CD10 in MCL could be attributed to morphological, immunophenotypic, and genetic characteristics, reflecting derivation from GC B cells.

The present study indicated that co-expression of CD10 and BCL-6 in MCL was associated with a shorter median survival time (12 months) and significantly lower rates of 1 -year OS and 1-year EFS $\left(\chi^{2}=6.401\right.$ and 5.975; $\mathrm{P}=0.011$ and 0.015 , respectively). However, the cox regression analysis indicated that patients with co-expression of CD10 and BCL-6 had no correlation with survival time $(\mathrm{P}=0.173)$. Several factors may cause poor prognosis of MCL patients with co-expression of CD10 and BCL-6. The results showed that WBC count, as well as the levels of LDH and $32-M G$ in MCL patients with CD10 and BCL6 co-expression were significantly higher than in those without CD10 and BCL-6 co-expression ( $\mathrm{P}=0.038,0.015$, and 0.021 , respectively). High levels of $\mathrm{LDH}$ and $\beta 2-\mathrm{MG}$, and a great number of WBCs mainly indicate DP or poor prognosis $(24,25)$. Cytogenetic factors were utilized in the study of a large number of hematologic malignancies, while they have been rarely applied in the assessment of MCL. Chromosomal instability, due to a disrupted DNA damage response, in conjunction with an abnormal activation of cell survival mechanism, underlies the aggressive clinical course in MCL (26). In 8 cases, according to the results of karyotyping, the $\mathrm{t}(11 ; 14)$ translocation was dominant, except for in 3 cases, while the presence of rearrangement was confirmed by FISH. Additionally, two cases had CKs ( $\geq 3$ chromosomal abnormalities), and additional abnormalities included chromosomes 1, 5, 7, 17, and 19 . The aberrant expressions of CD10 and BCL- 6 were found to be associated with cytogenetic abnormalities and a poor outcome $(27,28)$. Greenwell et al. (29) showed that a CK was associated with significantly reduced OS (11.6 vs. 4.5 years) and PFS (4.4 vs. 1.9 years) compared with nonCK in MCL patients, and suggested that cytogenetic factors should be considered in the diagnosis of MCL, and further novel therapeutic approaches should be presented for patients with CK. In smaller series, both a CK and specific cytogenetic abnormalities, such as $\operatorname{del}(17 \mathrm{p})$, have been found to be associated with inferior outcomes. A number of scholars (30) demonstrated that TP53 mutations occur 
in approximately $5-11 \%$ of MCL cases, while the result of TP53 genetic test was positive in $50 \%$ of patients with CD10 and BCL-6 co-expression in the current study. The positive result of TP 53 genetic test is mainly associated with a shorter OS. Therefore, it was reported that the change of 17P/TP53 may be one of the mechanisms of MCL progression (31). In the present study, the conventional cytogenetic analysis showed a high frequency of CK and TP53 abnormalities, whereas compared with the traditional prognostic markers of MIPI and Ki-67, the role of genetic aberrations in MCL still remained controversial.

In conclusion, co-expression of BCL-6 and CD10, although being rare in MCL, may lead to misdiagnosis. Compared with MCL patients without co-expression of CD10 and BCL-6, MCL patients with co-expression of CD10 and BCL-6 were more likely to have bone marrow involvement, higher Ki-67 index, increased WBC count, and elevated levels of $\mathrm{LDH}$ and $\beta 2-\mathrm{MG}$ at the time of diagnosis. It might depend on a variety of molecular mechanisms unrelated to GC events. Hence, further studies are required to elucidate the pathological and prognostic features of MCL patients with co-expression of CD10 and BCL-6.

\section{Acknowledgments}

We would like to thank Dr. Tingbo Liu who made great contribution to this study.

Funding: This work was supported by the National and Fujian Provincial Key Clinical Specialty Discipline Construction Program, China, Construction Project of Fujian Medical Center of Hematology (Min 201704), and the Foundation for Scientific Research Projects of Fujian Medical University (2016QH030).

\section{Footnote}

Reporting Checklist: The authors have completed the REMARK reporting checklist. Available at https://dx.doi. org/10.21037/tcr-21-882

Data Sharing Statement: Available at https://dx.doi. org/10.21037/tcr-21-882

Conflicts of Interest: All authors have completed the ICMJE uniform disclosure form (available at https://dx.doi. org/10.21037/tcr-21-882). The authors have no conflicts of interest to declare.
Ethical Statement: The authors are accountable for all aspects of the work in ensuring that questions related to the accuracy or integrity of any part of the work are appropriately investigated and resolved. The study was conducted in accordance with the Declaration of Helsinki (as revised in 2013). This study was approved by the Ethics Committee of the Fujian Medical University Union Hospital (approval No. 2019KY123) and individual consent for this retrospective analysis was waived.

Open Access Statement: This is an Open Access article distributed in accordance with the Creative Commons Attribution-NonCommercial-NoDerivs 4.0 International License (CC BY-NC-ND 4.0), which permits the noncommercial replication and distribution of the article with the strict proviso that no changes or edits are made and the original work is properly cited (including links to both the formal publication through the relevant DOI and the license). See: https://creativecommons.org/licenses/by-nc-nd/4.0/.

\section{References}

1. Choi SM, O'Malley DP. Diagnostically relevant updates to the 2017 WHO classification of lymphoid neoplasms. Ann Diagn Pathol 2018;37:67-74.

2. Condoluci A, Rossi D, Zucca E, et al. Toward a RiskTailored Therapeutic Policy in Mantle Cell Lymphoma. Curr Oncol Rep 2018;20:79.

3. Bosch F, Jares P, Campo E, et al. PRAD-1/cyclin D1 gene overexpression in chronic lymphoproliferative disorders: a highly specific marker of mantle cell lymphoma. Blood 1994;84:2726-32.

4. Jares P, Colomer D, Campo E. Genetic and molecular pathogenesis of mantle cell lymphoma: perspectives for new targeted therapeutics. Nat Rev Cancer 2007;7:750-62.

5. Dreyling M, Kluin-Nelemans HC, Beà S, et al. Update on the molecular pathogenesis and clinical treatment of mantle cell lymphoma: report of the 11th annual conference of the European Mantle Cell Lymphoma Network. Leuk Lymphoma 2013;54:699-707.

6. Jain P, Wang M. Mantle cell lymphoma: 2019 update on the diagnosis, pathogenesis, prognostication, and management. Am J Hematol 2019;94:710-25.

7. Goy A, Kalayoglu Besisik S, Drach J, et al. Longer-term follow-up and outcome by tumour cell proliferation rate (Ki-67) in patients with relapsed/refractory mantle cell lymphoma treated with lenalidomide on MCL-001(EMERGE) pivotal trial. Br J Haematol 
2015;170:496-503.

8. Swerdlow SH. International Agency for Research on Cancer. World Health Organization. WHO classification of tumours of haematopoietic and lymphoid tissues. 4th ed. Lyon, France: International Agency for Research on Cancer, 2008;439.

9. Maguer-Satta V, Besançon R, Bachelard-Cascales E. Concise review: neutral endopeptidase (CD10): a multifaceted environment actor in stem cells, physiological mechanisms, and cancer. Stem Cells 2011;29:389-96.

10. Basso K, Dalla-Favera R. Roles of BCL6 in normal and transformed germinal center B cells. Immunol Rev 2012;247:172-83.

11. Basso K, Dalla-Favera R. BCL6: master regulator of the germinal center reaction and key oncogene in B cell lymphomagenesis. Adv Immunol 2010;105:193-210.

12. Camacho FI, García JF, Cigudosa JC, et al. Aberrant Bcl6 protein expression in mantle cell lymphoma. Am J Surg Pathol 2004;28:1051-6.

13. Gao J, Peterson L, Nelson B, et al. Immunophenotypic variations in mantle cell lymphoma. Am J Clin Pathol 2009;132:699-706.

14. Akhter A, Mahe E, Street L, et al. CD10-positive mantle cell lymphoma: biologically distinct entity or an aberrant immunophenotype? Insight, through gene expression profile in a unique case series. J Clin Pathol 2015;68:844-8.

15. Oka K, Ohno T, Yamaguchi M, et al. PRAD1/Cyclin D1 gene overexpression in mantle cell lymphoma. Leuk Lymphoma 1996;21:37-42.

16. Tiemann M, Schrader C, Klapper W, et al. Histopathology, cell proliferation indices and clinical outcome in 304 patients with mantle cell lymphoma (MCL): a clinicopathological study from the European MCL Network. Br J Haematol 2005;131:29-38.

17. Li J, Hu CM, Ding XD, et al. Immunohistochemical analysis of 146 cases of mantle cell lymphoma. J Clin Pathol Res 2016;36:733-6.

18. Gualco G, Weiss LM, Harrington WJ Jr, et al. BCL6, MUM1, and CD10 expression in mantle cell lymphoma. Appl Immunohistochem Mol Morphol 2010;18:103-8.

19. Vardiman JW. The World Health Organization (WHO) classification of tumors of the hematopoietic and lymphoid tissues: an overview with emphasis on the myeloid neoplasms. Chem Biol Interact 2010;184:16-20.

20. Hashmi AA, Hussain ZF, Faridi N, et al. Distribution of Ki67 proliferative indices among WHO subtypes of nonHodgkin's lymphoma: association with other clinical parameters. Asian Pac J Cancer Prev 2014;15:8759-63.
21. Foshat M, Stewart J, Khoury JD, et al. Accuracy of diagnosing mantle cell lymphoma and identifying its variants on fine-needle aspiration biopsy. Cancer Cytopathol 2019;127:44-51.

22. Wasik AM, Priebe V, Lord M, et al. Flow cytometric analysis of SOX11: a new diagnostic method for distinguishing B-cell chronic lymphocytic leukemia/small lymphocytic lymphoma from mantle cell lymphoma. Leuk Lymphoma 2015;56:1425-31.

23. Zanetto U, Dong H, Huang Y, et al. Mantle cell lymphoma with aberrant expression of CD10. Histopathology 2008;53:20-9.

24. Ohanian M, Rozovski U, Ravandi F, et al. Very high levels of lactate dehydrogenase at diagnosis predict central nervous system relapse in acute promyelocytic leukaemia. Br J Haematol 2015;169:595-7.

25. Yoo C, Yoon DH, Suh C. Serum beta-2 microglobulin in malignant lymphomas: an old but powerful prognostic factor. Blood Res 2014;49:148-53.

26. Jares P, Colomer D, Campo E. Molecular pathogenesis of mantle cell lymphoma. J Clin Invest 2012;122:3416-23.

27. Sakhdari A, Ok CY, Patel KP, et al. TP53 mutations are common in mantle cell lymphoma, including the indolent leukemic non-nodal variant. Ann Diagn Pathol 2019; $41: 38-42$.

28. Clot G, Jares P, Giné E, et al. A gene signature that distinguishes conventional and leukemic nonnodal mantle cell lymphoma helps predict outcome. Blood 2018;132:413-22.

29. Greenwell IB, Staton AD, Lee MJ, et al. Complex karyotype in patients with mantle cell lymphoma predicts inferior survival and poor response to intensive induction therapy. Cancer 2018;124:2306-15.

30. Eskelund CW, Dahl C, Hansen JW, et al. TP53 mutations identify younger mantle cell lymphoma patients who do not benefit from intensive chemoimmunotherapy. Blood 2017;130:1903-10.

31. Cohen JB, Ruppert AS, Heerema NA, et al. Complex karyotype is associated with aggressive disease and shortened progression-free survival in patients with newly diagnosed mantle cell lymphoma. Clin Lymphoma Myeloma Leuk 2015;15:278-85.e1.

Cite this article as: Chen W, Liu H, Wang P, Li G. Clinical and biological features of mantle cell lymphoma patients with co-expression of CD10 and BCL-6: a retrospective study. Transl Cancer Res 2021;10(11):4786-4793. doi: 10.21037/tcr-21-882 